\title{
PARTICLE ACCELERATION ON THE SUN AND IN THE HELIOSPHERE
}

\author{
E. C. Roelof \\ Johns Hopkins University/Applied Physics Laboratory, USA
}

\begin{abstract}
Examples of the acceleration of energetic ions and electrons are given for the Sun, the middle heliosphere, and the outer heliosphere. The characteristics of the acceleration are revealed only after the effects of energetic particle propagation are understood.
\end{abstract}

\section{Introduction}

We need to understand the mechanisms controlling the propagation of energetic particles throughout the heliosphere before we can analyze their acceleration processes. Two transport mechanisms that are always operative are field-aligned transport and particle energy loss. A third mechanism is transport transverse to the magnetic field (in addition to the deterministic convection of the guiding center of the particles along with the field lines that are "frozen" into the solar wind) depends on the degree to which heliospheric field lines are "braided" or "random walk." Field-aligned transport results from weak pitch-angle scattering combined with the focussing effect of the outwardly decreasing interplanetary magnetic field (IMF). A species-independent fractional momentum loss rate in this weak-scattering limit is produced by the magnetic field gradient and curvature drifts of the particles having a component anti-parallel to the electric field in the solar wind. We shall present some recent results that bear on relevant aspects of energetic particle acceleration and transport in the three different regions: the Sun, and the Inner, Middle, and Outer Heliosphere.

\section{The Sun and Inner Heliosphere}

In impulsive solar energetic particle (SEP) events, relativistic protons and electrons observed at $1 \mathrm{AU}$ often reach maximum intensity within tens of minutes after the event onset. The onset itself follows the electromagnetic emission associated with the event by approximately the transit times of the particles. Evidence has mounted over the years that the actual injection times for nearrelativistic particles is actually delayed several minutes after the prompt electromagnetic emission (gamma ray, $\mathrm{x}$-ray, and micro wave radio bursts). We will touch briefly on this important point at the end of this section. Lower energy (non-relativistic) particles take much longer to rise to their maximum intensity in impulsive events, but their onset time is still close to the time of the associated electromagnetic emission (after subtraction of their transit time from the 

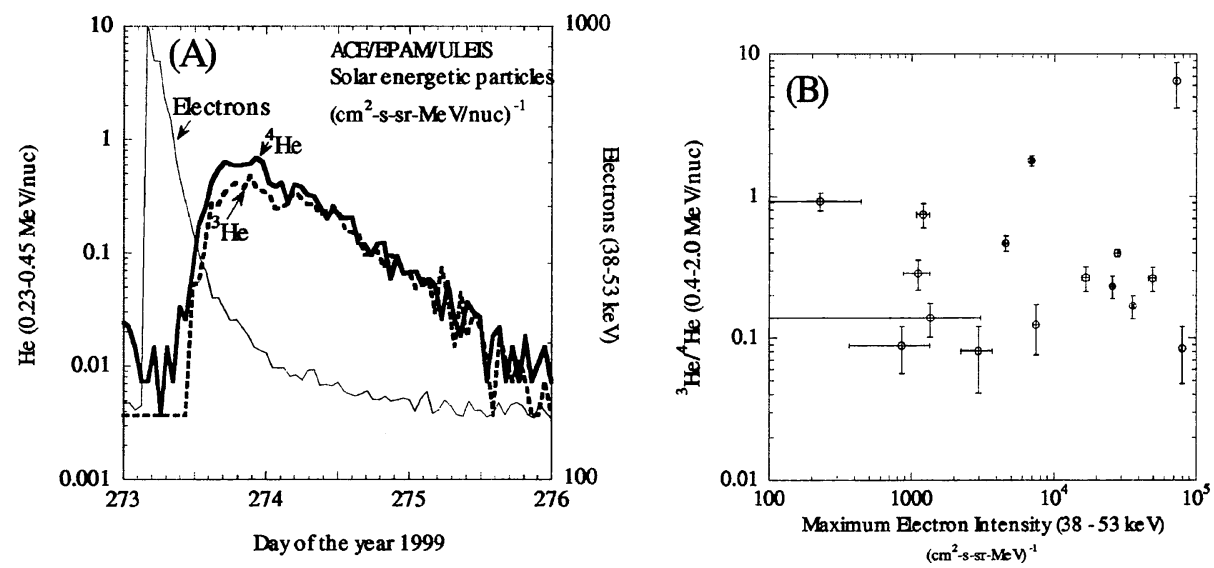

Figure 1. An example of an impulsive electron-associated ${ }^{3} \mathrm{He}$-rich event 30 September 1999 [Ho et al. 2001]: (A) intensity histories; (B) lack of correlation between event-averaged ${ }^{3} \mathrm{He} /{ }^{4} \mathrm{He}$ ratio and maximum electron intensities.

Sun). Therefore acceleration must therefore have commenced within a few solar radii of the Sun.

An example of an interesting subset of SEP events is presented in Figure 1a: near-relativistic (38-53 keV) electrons, and the two isotopes ${ }^{3} \mathrm{He}$ and ${ }^{4} \mathrm{He}$ (0.23-0.45 MeV/nuc). The analysis is by Ho et al. (2001) utilized data from the EPAM (Gold et al., 1998) and ULEIS (Mason et al., 1998) instruments on the ACE spacecraft located in a Lagrangian L1 orbit $\sim 235 R_{E}$ sunward of the Earth. A property of impulsive SEP events, of which this event from 29 September 1998 is typical, is that the histories are very similar when expressed in units of the distance traveled (vt) at the particle velocity (v). This can readily be seen in Fig. 1a, because a $45 \mathrm{keV}$ electron $(\mathrm{v} / \mathrm{c}=0.40)$ takes 21 minutes to travel $1 \mathrm{AU}$, while $0.32 \mathrm{MeV} \mathrm{nuc}{ }^{-1} \mathrm{He}$ ion $(\mathrm{v} / \mathrm{c}=0.026)$ takes 5.3 hours. The rise-to-maximum time for each species is a couple of transit times, and the duration of each event (measured at $10 \%$ of the maximum) is about a half-a-dozen transit times in both cases. These impulsive SEPs usually exhibit pitch-angle distributions (PADs) that are strongly collimated outward along the magnetic field during the rise to maximum. Lin (1970) demonstrated that $\sim 50 \mathrm{keV}$ electrons propagated approximately "scatter-free," and later Potter et al. (1980) showed that the same was true down to energies $\sim 2 \mathrm{keV}$. We call these events "beam-like."

The importance of the beam events follows from the fact that the particles must have traveled most of the distance from the sun at a very small pitch angle. Then their transit times are actually well-approximated by $\mathrm{z} / \mathrm{v}$, where $\mathrm{z}=1.2 \mathrm{AU}$ for a nominal Parker spiral magnetic field at $1 \mathrm{AU}$. This means that during the intensity rise to maximum (while the PADs are beam-like) we are essentially observing the injection history of the particles at the Sun, delayed in time by the approximate transit time $\mathrm{z} / \mathrm{v}$. For near-relativistic electrons, there is a complication. The electrons undergo wave/particle interactions en route to $1 \mathrm{AU}$, probably generating Langmuir waves by inverse Landau damping in the 
solar wind (Roelof, 2000a). However, these interactions are readily identified by the appearance of a flat energy spectrum during the early rise phase, and they do not obscure the actual injection history during the final portion of the rise to maximum.

Because of the scatter-free propagation, the maximum intensity should be proportional to that released from the Sun, and the constant of proportionality $(\sim \exp (-\mathrm{z} / \lambda)$ should not vary greatly from one event to another. This fact allows us to test hypotheses concerning the role that energetic electrons play in solar acceleration processes. For example, Temerin and Roth (1992) and Roth and Temerin (1997) proposed, by analogy to electron acceleration processes observed above the Earth's auroral zones, that electron ion cyclotron (EMIC) waves generated by beams of energetic electrons could accelerate heavy ions. They applied the theory to the puzzling extraordinary enhancements in the ${ }^{3} \mathrm{He} /{ }^{4} \mathrm{He}$ ratio observed in SEP events (Hsieh and Simpson 1970; Mason et al. 1986). They were motivated by the result that ${ }^{3} \mathrm{He}$-rich events were usually accompanied by energetic electrons (Reames et al. 1985). The ${ }^{3} \mathrm{He} /{ }^{4} \mathrm{He}$ ratio is $\sim 10^{-4}$ in the thermal solar wind (and therefore presumably also the same in typical coronal plasma), but can exceed unity at helium ion energies $\sim 1 \mathrm{MeV} /$ nuc. The electron-associated He event shown in Fig. 1 has a ratio ${ }^{3} \mathrm{He} /{ }^{4} \mathrm{He} \sim 1$.

It therefore came as a surprise when Ho et al. (2000) analyzed $27{ }^{3} \mathrm{He}$-rich impulsive SEP events observed by ACE/ULEIS/EPAM during 1997-1999 and found that, while 16 out of the 27 were associated with impulsive beam-like $45 \mathrm{keV}$ electron events, there was no significant correlation between the eventaveraged ${ }^{3} \mathrm{He} /{ }^{4} \mathrm{He}$ ratio and the maximum electron intensity (see Figure $1 \mathrm{~b}$ ). They concluded that the $40-300 \mathrm{keV}$ electrons that were injected into the solar wind were not involved in the preferential acceleration of ${ }^{3} \mathrm{He}$. Furthermore, they showed that the degree of association of impulsive electron events with ${ }^{3} \mathrm{He}$-rich events was statistically the same as their association with $\mathrm{He}$ events in general. These results reinforce the renewed conviction in the hypothesis originally proposed by Wild et al. (1963) that the impulsive energetic particle populations we measure in interplanetary space are accelerated by shocks traveling out through the corona (see the recent review by Cliver 2000). That is why the injection of the near-relativistic interplanetary particles is delayed by several minutes relative to the prompt electromagnetic emission. The latter is produced by a particle population that does not escape the Sun, while the former is accelerated by a shock with a velocity $\sim 1000 \mathrm{~km} / \mathrm{s}$ that moves 1 solar radius every 12 minutes (thus explaining the observed delays $\sim 10$ minutes).

\section{The Middle Heliosphere}

During the first southern pass of the Ulysses spacecraft there was a remarkable sequence of 26-day recurrent energetic particle events all the way up to the maximum latitude attained (80.22S heliographic on 14 September 1994. Electrons with energies $50 \mathrm{keV}$ could be tracked right through the highest latitudes (Roelof et al. 1996) and protons with energies $\sim 1 \mathrm{MeV}$ almost that high (Sanderson et al. 1996). At low to moderate latitudes, the energetic electron and ion events were clearly associated with corotating interaction regions, or CIRs (Sanderson et al. 1994; Simnett et al. 1994). These are stream-stream interactions in which 
the low-speed solar wind (from the streamer belt) interacts with high-speed solar wind (from the equatorial extension of polar coronal holes) to compress the interplanetary magnetic field (IMF), bounded by a forward shock (FS) at the leading edge and a reverse shock (RS) at the trailing edge (Smith and Wolfe 1996). However, the CIR shocks extended only to mid-latitudes (Gosling et al. 1993). Figure 2 shows two 26-day plots (one sidereal solar rotation) of recurrent events measured by the Ulysses HI-SCALE detectors (Lanzerotti et al. 1992) at radial distances of $5.0 \mathrm{AU}$ and $4.3 \mathrm{AU}$, respectively. The particle energy channels plotted are ions (68-115 keV, 321-587 keV, 1.9-4.8 MeV) and electrons (38-53 $\mathrm{keV})$. They are labeled by their velocities $(\beta=\mathrm{v} / \mathrm{c})$, assuming that the ions are predominantly protons.

The event presented in Fig. $2 \mathrm{a}$ is the ninth recurrence in the series seen by Ulysses, and is associated with a fully developed CIR at low latitudes (S24 and 4.3 $\mathrm{AU}$ ). Both the FS and the RS are present, and there are ion intensity enhancements at all energies closely associated with both (although there is none in the electrons at the FS). Fig. 2b shows a later, more southern recurrence (CIR 18 at $\mathrm{S}^{\circ} 9^{\circ}$ and $4.3 \mathrm{AU}$ ) on which only a RS was observed. The ion enhancements are still associated with the RS, although at the highest ion energy plotted (1.9$4.8 \mathrm{MeV}, \beta=0.08)$, the peak is clearly after the RS and is followed by a slower decay than in CIR 9. The same trend is seen in the electron history, but much more exaggerated. As Ulysses continued toward the southern heliospheric pole, the reverse shocks died out by $\mathrm{S} 46^{\circ}$ (Gosling et al. 1993). Yet the recurrent electron events continued to reappear every 26 days right up to the highest latitude achieved $\left(\mathrm{S} 80.1^{\circ}\right)$, long after the in situ shock signatures had ceased to appear. The lowest energy ions, however, dropped below instrument background well before the Ulysses' furthest south. Since the CIRs were the only likely acceleration site in the outer heliosphere for the recurrent electrons and ions, these energetic particles must reach Ulysses by following field lines from the CIRs at lower latitudes.

Simnett and Roelof (1995) argued that Ulysses at mid-latitudes had to be connected magnetically to the CIRs at helio-radii well beyond that of Ulysses. However, as the recurrent events continued to be observed up to high latitudes, it became clear that an ideal Parker magnetic field could not do this, because its field lines lie on cones of constant helio-latitude. Thus motivated to explain these high latitude recurrent energetic particle events, Fisk (1996) produced a new model of the heliospheric magnetic field based on photospheric differential rotation and non-radial motion of the near-sun solar wind. It predicts that some high-latitude field lines from the Sun will connect to CIRs at latitudes as low as $30^{\circ}$ at distances $\sim 15$ AU. Later, Kota and Jokipii (1999) argued that Parker field lines that wandered stochastically in latitude could accomplish the same connection and also explain the Ulysses energetic particle observations.

What remained to be explained was the ordering of the intensity histories by particle velocity that is evident in Fig. 2. Roelof (2000) argued that it was the direct result of a new understanding of the particle energy loss in the heliosphere. Based on the guiding center theory developed by Northrop (1963), Roelof showed that the instantaneous loss rate of particle momentum (p) in a Parker (or Fisk) magnetic field (for $r>2 \mathrm{AU}$ ) was given to good approximation by the simple expression $\operatorname{dlogp} / \mathrm{dt}=-\left(1+\mu^{2}\right)(\mathrm{V} / 2 \mathrm{r})$, where $\mathrm{V}$ is the solar wind 

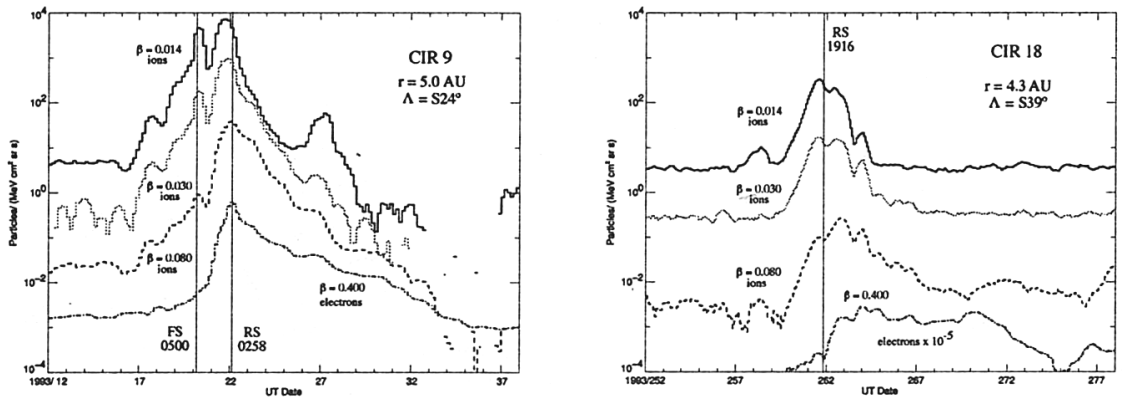

Figure 2. Intensity histories (labeled by velocities $(\beta=\mathrm{v} / \mathrm{c})$ of lowenergy ions and near-relativistic electrons for two 26-day recurrent CIRassociated particle events observed by Ulysses/HI-SCALE en route to the South pole in 1993.

and $\mu$ is the particle's pitch cosine. It is remarkable that this fractional loss rate is independent of the particle's mass, charge, or velocity; it also holds for relativistic particles. It then follows that the net fractional energy loss for particles travelling the same distance will depend only upon on their velocities. This is because they both lose momentum (or energy) at the same instantaneous fractional rate, so the faster particle loses less momentum because it covers the distance in a shorter time. The situation of propagation from the RS of a lowlatitude CIR at $15 \mathrm{AU}$ to Ulysses at $3 \mathrm{AU}$ at high latitude is sketched in Fig. 3. Electrons with $\mathbf{v}=0.4 \mathrm{c}$ will lose relatively little energy in transit (according to the formula), so Ulysses can receive electrons accelerated from the CIR for many days as it rotates under the spacecraft. The magnetic connection point on the RS of the CIR moves outward in radius with time, so the electrons are coming from progressively further distances as time increases. This explains both the delayed maximum and the long, slow decay of the electron intensities. On the other hand, the low energy ions with $v=0.014 c$ (only $1 / 300$ the speed of the electrons), will lose a large fraction of their energy. This is because it takes them 10 hours to travel $1 \mathrm{AU}$ along the field line from $15 \mathrm{AU}$ inward to $3 \mathrm{AU}$ (a distance along a Parker spiral of about $100 \mathrm{AU}$ ). The total transit would take 40 days; they had to have left the CIR on the previous solar rotation!

Consequently, they are observable at Ulysses only during the time of the shortest possible magnetic connection, and they will disappear below instrument background as the optimum connection distance lengthens with increasing Ulysses latitude. Thus the energy loss process, combined with the geometry shown in Fig. 3, can explain the velocity-ordered pattern of recurrent particle histories shown in Fig. 2.

\section{The Outer Heliosphere}

The outer heliosphere is populated with energetic particles that enter it from beyond the termination shock (thought to be located at $\mathrm{r} \sim 100 \mathrm{AU}$ ), such as galactic cosmic rays, and ions thought to be accelerated in the vicinity of the 


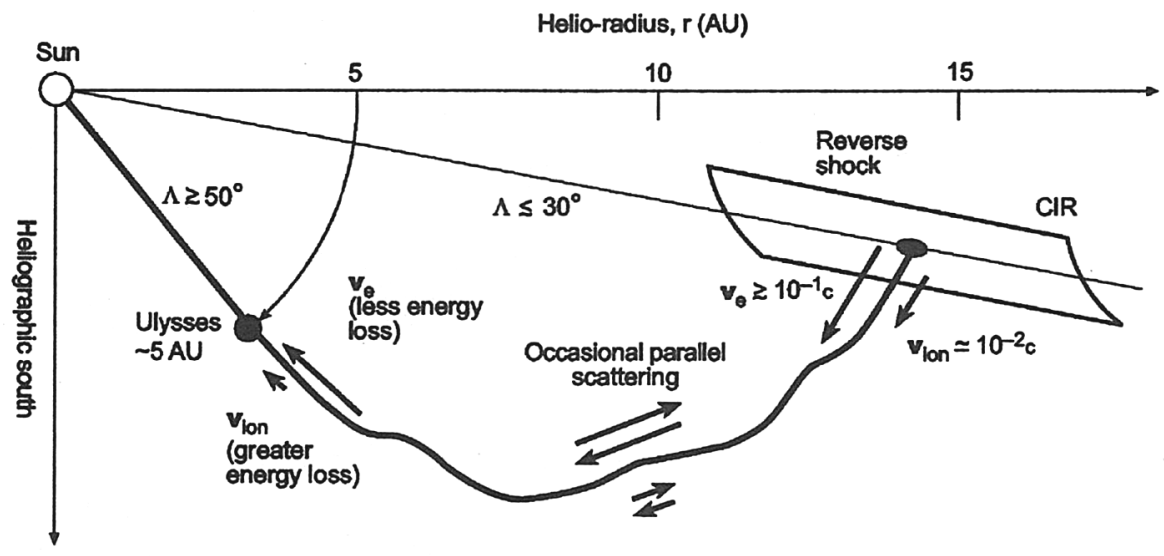

Figure 3. Effect of energy loss on the propagation of electrons and ions along magnetic field lines from CIRs at low latitudes to Ulysses at high latitudes [Roelof 2000].

termination shock, the "anomalous" cosmic rays with energies $\sim 100 \mathrm{MeV} /$ nuc. On rare occasions, particles of much lower energies $(\sim 1 \mathrm{MeV})$ that are accelerated by great solar events (SEPs) can survive the effects of energy loss and be observed by the Voyager $1 / 2$ spacecraft at distances $r>50$ AU. That happened in late 1998 following a sequence of solar flares and CMEs that produced a large SEP event at Earth on 20 April. Figure 4 (after Decker et al., 2000) depicts the arrival almost half a year later of these $0.5-1.5 \mathrm{MeV}$ protons at Voyager 2 $\left(55 \mathrm{AU}, \mathrm{S}^{\circ} 9^{\circ}\right)$ and Voyager $1\left(70 \mathrm{AU}, \mathrm{N}^{\circ} 3^{\circ}\right.$. Because of the very low absolute intensities, 5-day averages are plotted, along with their Poisson-statistical error bars. Also shown is are the intensities of $22-30 \mathrm{MeV}$ ACR protons. Independent measurements of ACR $\mathrm{H}$ and $\mathrm{He}$ have been analyzed by McDonald et al. (2000). About this same time, a very extended global merged interaction region (GMIR) also arrived and took about 50 days to pass over the spacecraft Burlaga and Ness 2000).

Decker et al. (2000) point out the remarkably similar histories of the $\sim 1$ $\mathrm{MeV}$ protons at the two Voyager spacecraft, even though they are separated by a total distance of some $70 \mathrm{AU}$. Moreover, the $\sim 1 \mathrm{MeV}$ proton histories bear the same relationship to the abrupt decrease in the 22-30 MeV ACR protons (indicated by the dashed line). They interpret the $\sim 1 \mathrm{MeV}$ increases as being due to the merging of the SEP populations produced by the series of solar disturbances in early April. These protons were observed for some 10 days by the Ulysses HI-SCALE ion detectors at equatorial latitudes (S7 ${ }^{\circ}$ ) at 5.4 AU. Their Gaussian-like shape with a total width of almost 90 days was explained by Decker et al. (2000) in terms of weak-scattering. The scattering must be weak along the magnetic field (i.e., scatter-free over distances $\sim 1 \mathrm{AU}$ ), because otherwise, the protons would lose too much energy in getting from $5 \mathrm{AU}$ to beyond $50 \mathrm{AU}$ (by the mechanism discussed in the previous section). Decker et 

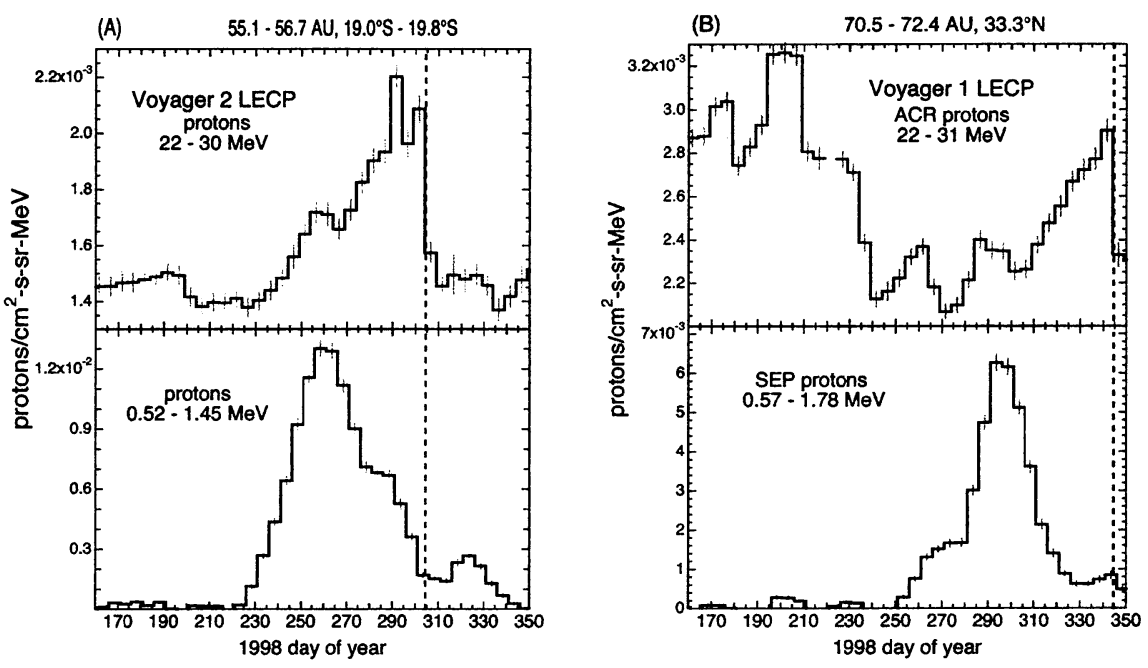

Figure 4. Behavior at 50-70 AU of energetic protons $\sim 1 \mathrm{MeV}$ accelerated by the solar events of April 1998 and their relation to abrupt decreases in 22-30 MeV ACR protons (Decker et al. 2000)

al. (2000) take the abrupt drop in the ACR protons as marking the arrival of the separatrix between the major solar wind disturbance and the undisturbed solar wind. This identification is not inconsistent with the solar wind measurements on Voyager 2; there is no active solar wind instrument of Voyager 1.

The interpretation is that the solar protons initially streamed outward along the magnetic field lines, away from the solar wind disturbances. Initially, they could outrun the disturbed solar wind, because their velocity is about 14,000 $\mathrm{km} \mathrm{s}^{-1}$. However, what counts in outrunning the solar wind is their net radial velocity. This diminishes rapidly because of the winding up of the spiral field. Consequently, even though they still may have a high velocity parallel to the magnetic field, as they work their way out to tens of AU from the Sun, their radial is reduced to only that of the solar wind. From then on, they "coast" ahead of the solar wind disturbance, having built up a radial lead in the race, but being spread over one or two windings of the field. Decker et al. (2000) calculate the lead distance to be about $12 \mathrm{AU}$, so that the peak in the intensity distribution should arrive at the Voyagers about 45 days prior to the marker of the disturbed solar wind (that they left behind in the inner heliosphere). The energy loss process is quit weak in the outer heliosphere, having a time constant $\sim(3 / 4) \mathrm{r} / \mathrm{V}=(3$ days/AU) $\mathrm{r}(\mathrm{AU}) \sim 150$ days at $50 \mathrm{AU}$. The $1 \mathrm{MeV}$ protons at the Voyagers would have left the inner heliosphere with energies $\sim 3-5 \mathrm{MeV}$.

Although this may explain the behavior of the solar particles, the mechanism for the abrupt decrease in the $22-30 \mathrm{MeV}$ is a separate (un-answered) question. Nonetheless, the juxtaposition of the behaviors of these distinct populations in the outer heliosphere demands a consistent explanation. This example again illustrates the necessity for understanding energetic particle propagation in order to get at the question of energetic particle acceleration. 


\section{Summary}

A diversity of energetic particle acceleration processes operates throughout the heliosphere, from the Sun to the outer boundary 100 AU. Because these particles travel so easily throughout the heliosphere, the characteristics of their acceleration can only be deduced when we properly understand the nature of their global propagation.

Acknowledgments. This work was supported by Voyager Interstellar Mission under,NASA Grant NAG5-4365, by Ulysses Hi-SCALE under NASA Grant NAG5-6113, and by ACE EPAM under NASA Contract NAS5-97271.

\section{References}

Burlaga, L.F. \& Ness, N.F. 2000, J. Geophys. Res., 105, 5142

Cliver, E.W. 2000, AIP Conf. Proc., 528, 21

Decker, R.B., Roelof, E.C., and Krimigis, S.M. 2000, AIP Conf. Proc., 528, 161

Fisk, L. A. 1996, J. Geophys. Res., 101, 15547

Gold, R. E. et al. 1998, Space Sci. Rev., 86, 541

Gosling, J. T. et al. 1993, Geophys. Res. Lett., 20, 2789

Ho, G. et al. 2001, ApJ, in press

Hsieh, K. C. \& Simpson, J. A. 1970, ApJ, 162, L191

Kota, J. \& Jokipii, J. R. 1999, Space Sci. Rev., 89, 240

Krimigis, S. M. et al. 1977, Space Sci. Rev., 21, 329

Lanzerotti, L. J. et al. 1992, A \& A, 92, 349

Lin, R. P., 1970, J. Geophys. Res., 75, 2583

McDonald, F. B. et al. 2000, J. Geophys. Res. 105, 20997

Mason, G. M. et al. 1986, ApJ, 303, 849

Mason, G. M. et al. 1998, Space Sci. Rev., 86, 409

Northrop, T. G. 1963, The Adiabatic Motion of Charged Particles, Interscience Publishers (John Wiley \& Sons), New York

Potter, D. W., Lin, R. P. \& Anderson, K. A. 1980, ApJ, L97

Reames, D. V., von Rosenvinge, T., \& R. P. Lin 1985, ApJ, 292, 716

Roelof, E. C., Simnett, G. M. \& Tappin, S. J., 1996, A\&A, 316, 481

Roelof, E. C. 1999, Space Sci. Rev., 89, 238

Roelof, E. C. 2000a, EOS, Trans. AGU Supplement, 81, F953

Roelof, E. C. 2000b, AIP Conf. Proc., 528, 242

Roth, I. \& Temerin, M. 1997, ApJ, 477, 940

Sanderson, T. R. et al. 1996, in Solar Wind 8, AIP Conf. Proc., 411

Sanderson, T. R. et al. 1994, Geophys. Res. Lett., 21, 1113

Simnett, G. M. \& Roelof, E. C. 1995, Space Sci. Rev., 72, 303

Simnett et al. 1994, Geophys. Res. Lett., 21, 1561

Smith, E. J. \& Wolfe, J.H., Geophys. Res. Lett., 3, 137

Temerin, M. \& Roth, I. 1992, ApJ, 391, L105

Wild, J. P., Smerd, S. F., \& Weiss, A. A. 1963, ARA\&A, 1, 291 